Review

\title{
DAZ Family Proteins, Key Players for Germ Cell Development
}

\author{
Xia-Fei Fu 1,2,\#, Shun-Feng Cheng 1,3,\#, Lin-Qing Wang 1,3, Shen Yin 1,3, Massimo De Felici ${ }^{4,}$, Wei Shen $1,3, \bowtie$ \\ 1. Institute of Reproductive Sciences, Qingdao Agricultural University, Qingdao 266109, China \\ 2. College of Life Sciences, Qingdao Agricultural University, Qingdao 266109, China \\ 3. Key Laboratory of Animal Reproduction and Germplasm Enhancement in Universities of Shandong, College of Animal Science and Technology, \\ Qingdao Agricultural University, Qingdao 266109, China \\ 4. Department of Biomedicine and Prevention, University of Rome 'Tor Vergata', Rome 00133, Italy \\ \# Co-first authors \\ $\triangle$ Corresponding authors: Prof. Wei Shen, College of Animal Science and Technology, Qingdao Agricultural University, Qingdao, 266109, China. \\ E-mail: wshen@qau.edu.cn or Prof. Massimo De Felici, Department of Biomedicine and Prevention, University of Rome 'Tor Vergata', Rome 00133, \\ Italy. E-mail: defelici@uniroma2.it
}

(C) 2015 Ivyspring International Publisher. Reproduction is permitted for personal, noncommercial use, provided that the article is in whole, unmodified, and properly cited. See http://ivyspring.com/terms for terms and conditions.

Received: 2015.01.09; Accepted: 2015.07.23; Published: 2015.08.15

\begin{abstract}
DAZ family proteins are found almost exclusively in germ cells in distant animal species. Deletion or mutations of their encoding genes usually severely impair either oogenesis or spermatogenesis or both. The family includes Boule (or Boll), Dazl (or Dazla) and DAZ genes. Boule and Dazl are situated on autosomes while $D A Z$, exclusive of higher primates, is located on the $Y$ chromosome. Deletion of $D A Z$ gene is the most common causes of infertility in humans. These genes, encoding for RNA binding proteins, contain a highly conserved RNA recognition motif and at least one DAZ repeat encoding for a 24 amino acids sequence able to bind other mRNA binding proteins. Basically, Daz family proteins function as adaptors for target mRNA transport and activators of their translation. In some invertebrate species, BOULE protein play a pivotal role in germline specification and a conserved regulatory role in meiosis. Depending on the species, DAZL is expressed in primordial germ cells (PGCs) and/or pre-meiotic and meiotic germ cells of both sexes. Daz is found in fetal gonocytes, spermatogonia and spermatocytes of adult testes. Here we discuss DAZ family genes in a phylogenic perspective, focusing on the common and distinct features of these genes, and their pivotal roles during gametogenesis evolved during evolution.
\end{abstract}

Key words: Dazl; DAZ; Boule; germ cell; meiosis

\section{Introduction}

DAZ (Deleted in Azoospermia) family genes are important fertility factors in many animals including humans. This family includes three members, Boule (or Boll), Dazl (or Dazla) and DAZ, encoding RNA binding proteins. DAZ family genes are highly conserved. Ancestral Boule is present from sea anemones through humans and Dazl is conserved among vertebrates. However, DAZ is present only in higher primates, and no DAZ homolog is found in unicellular organisms.

In all species, Boule and Dazl are located in single copy on autosomes, while multiple $D A Z$ genes are located on the $\mathrm{Y}$ chromosome. DAZ is produced by the transposition and amplification of Dazl during primate evolution while Boule is considered as the grandfather of the family. In human, an array of four $D A Z$ genes (DAZ 1-4) is located in two clusters on the $\mathrm{Y}$ chromosome and mutations of these genes cause severe oligospermia or azospermia [1].

DAZ family proteins are located in the nucleus and/or in the cytoplasm of male and female germ cells at different developmental stages throughout the gametogenesis. The localization of DAZ family proteins suggests that they can regulate mRNA transla- 
tion occurring in the cytoplasm. These proteins have a highly conserved RNA recognition motif (RRM) for binding target mRNAs and at least one characteristic sequence of 24 amino acids, which are termed as DAZ repeats [1]. Dazl can regulate the expression, transport and localization of target mRNAs of proteins which control the differentiation, growth and maturation of germ cells. Combining with other proteins, DAZ family members play a crucial role in male and female gametogenesis conserved throughout the evolution. Basically, DAZ family proteins have been proposed to function as adaptors for mRNA transport and activators of their translation. In the present paper, we will review the current information about the molecular characteristics, the expression and functions of the $D A Z$ family genes and proteins during germ cell development in several animal species.

\section{The localization of $D A Z$ family genes}

$D A Z$ family genes are expressed almost exclusively in the germ cells. In the human genome, three members of DAZ family have been identified: Boule, Dazl and DAZ. Boule is located on chromosome 2 as a single copy. Dazl is on chromosome 3 , also as in single copy. Palindromic_duplications of $D A Z$ are found on the $Y$ chromosome as two clusters of four genes (DAZ 1-4) [1]. From the evolutionary perspective, Boule is an ancestral gene conserved from sea anemones to humans. The other two members of DAZ family evolved from Boule. Dazl appears in early vertebrate while $D A Z$ comes into the $\mathrm{Y}$ chromosome in the primates about 30 - 40 million years ago, by translocation and amplification of existing autosomal Dazl [2,3]. The DAZ transcription unit appears to contain at least 16 exons and to span about $42 \mathrm{~kb}$. Exon 1 consists of the initiator codon, exons 2 - 6 encode the RNA-binding domain, and exon 7 encodes a variable number of DAZ repeats. There are several variations in the sequence of the $D A Z$ repeat. Each copy of the gene also contains a $10.8 \mathrm{~kb}$ region that may be amplified. This region includes five exons that encode an RRM domain. In the human male, Dazl and DAZ sequences share a similarity of about $90 \%$ [2].

According to their sequence, expression and types of germ cells in which they are expressed, the $D A Z$ family genes can be divided into Dazl and Boule subfamilies. The first subfamily, including $D A Z$ and Dazl. Dazl is required throughout germ cell development and $D A Z$ is functional in male germ cells such as the prospermatogonia/gonocytes, spermatogonia, spermatocytes and elongated spermatids. In most species, Boule is expressed in meiotic cells, while in some invertebrates it is found in the germline founders [4].

\section{Structural characteristics of the DAZ family proteins}

DAZ family proteins contain an RRM and at least one DAZ repeat of 24 amino acids rich in Asn, Tyr, and Gln residues (Fig. 1; Table 1). Only DAZ proteins contain 9 to $15 \mathrm{DAZ}$ repeats. Male primates contain multiple $D A Z$ genes with varying numbers of polymorphic DAZ repeats [5-7]. The RRM motif is highly conserved and binds mRNAs at the untranslated regions (UTRs) [8], while in some cases, the DAZ motif is thought to mediate interaction with others proteins [9]. DAZL and BOULE proteins contain a single RRM and a single copy of DAZ motif. The RRM-type RNA-binding domain contains two conserved motifs called RNP-2 and RNP-1, corresponding to beta- 1 and beta- 3 sheets, respectively. Among the three proteins, DAZL and DAZ are highly homologous. The sequence similarity of Dazl in mouse and human is around 85\% [2]. The homology of Boule with the other two proteins is around $50-60 \%$. On the other hand, the Boule of mouse and human are similar to those of invertebrates.

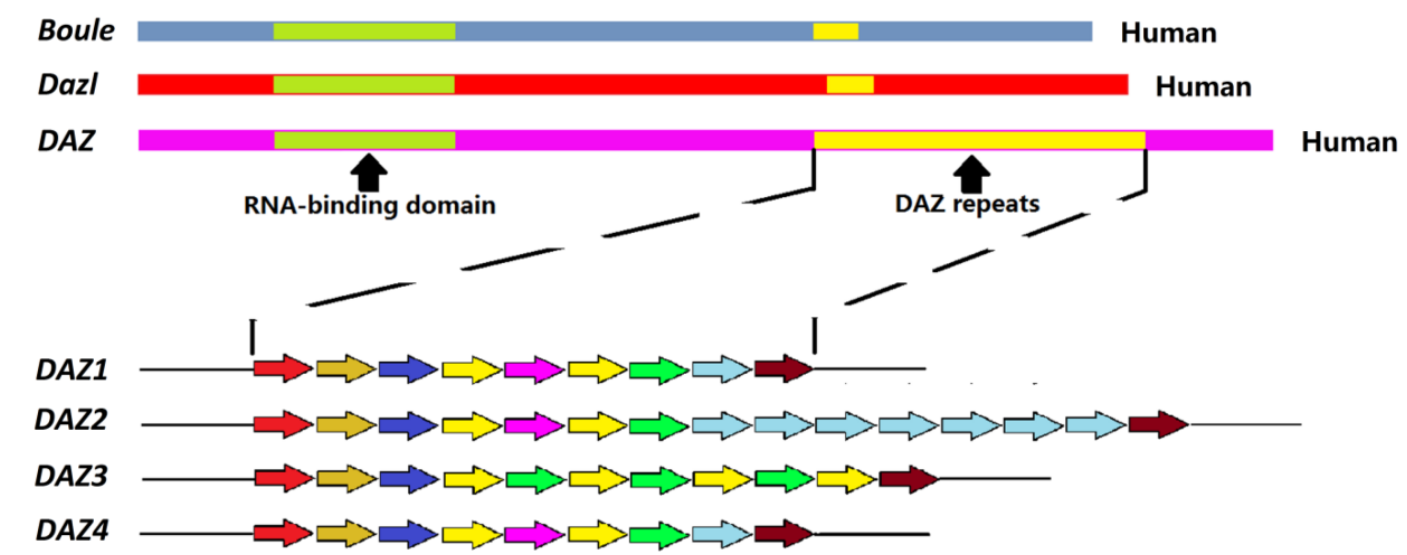

Figure 1. Schematic representation of the structure of the human $D A Z$ genes. Up: diagram of Boule, Dazl, and DAZ genes displaying the RNA-binding domains (green) and the DAZ repeats (yellow). Down: Different DAZI-4 repeats; the colors indicate different repeat sequence. 
Table 1. DAZ repeat sequences.

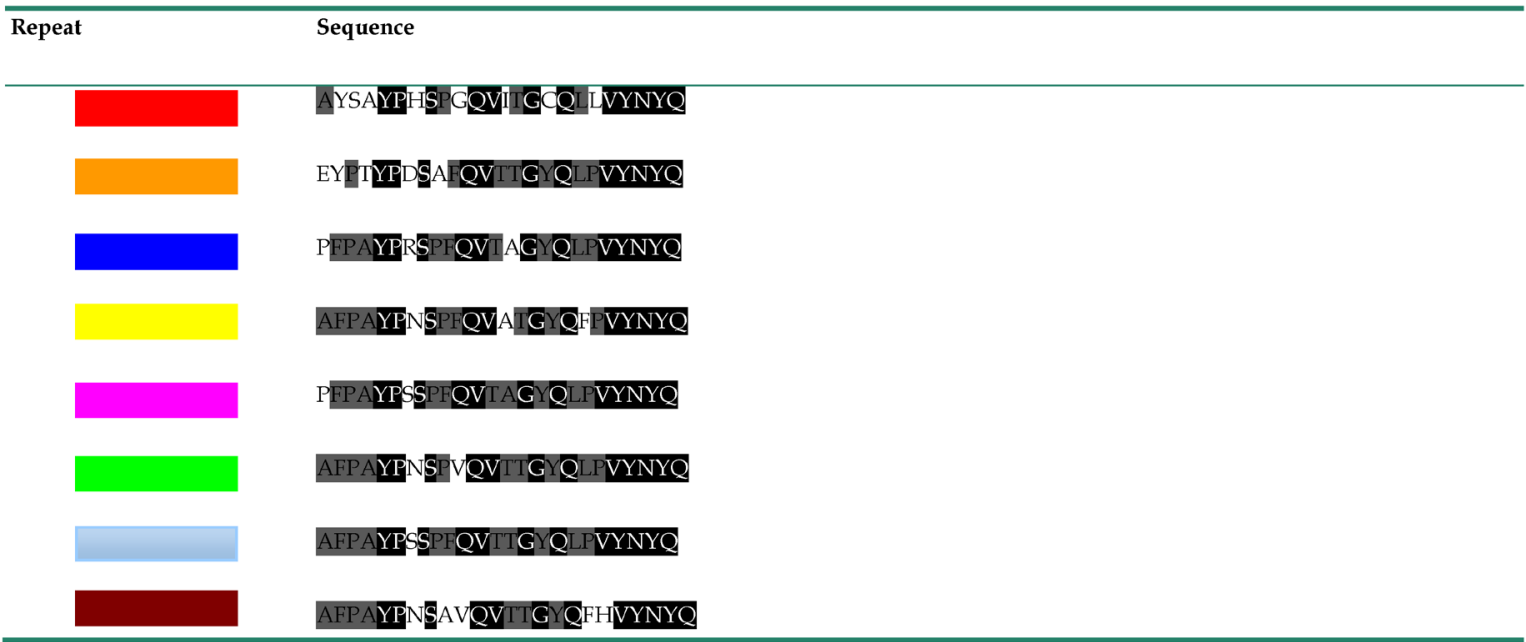

Note: The left column indicates the color repeat found in Fig. 1. The right-hand column indicates the sequence for each of the repeats. Black background indicates completely conserved amino acid residues. Gray background indicates highly conserved amino acid residues.

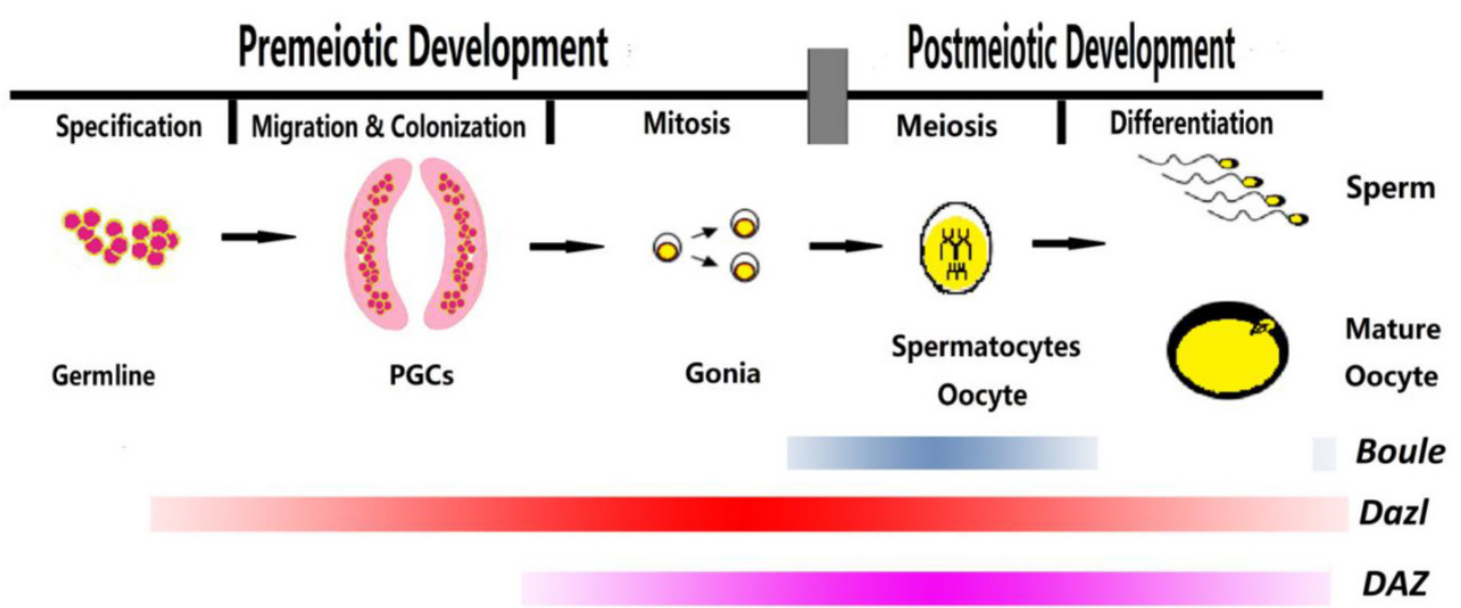

Figure 2. Expression profiles of Boule, Dazl, and DAZ genes during human gametogenesis.

\section{Space-temporal expression of the DAZ family genes and functions of the en- coded proteins}

In different species, DAZ family genes are expressed at different stages in diverse types of germ cells during female and male gametogenesis (Fig. 2). Nevertheless, it is possible to find some common principles driving the expression and functions of the encoded proteins. DAZL is generally pivotal in the capability of germ cells to sex differentiate either in meiotic oocytes or prospermatogonia [10-13]. At the same time, it is involved in the meiotic prophase I progression and maturation of oocytes [14, 15] and male germ cells [16-18]. The fact that Dazl is expressed in human and mouse pluripotent embryonic stem cells (ESCs) gains support to a conserved role of the proteins in germline determination underlying latent pluripotency. Actually, BOULE or its homologues are involved in the germline specification in some invertebrate species and play their functions during the mid-late phase of the meiotic prophase in both sexes of most species [19-21]. Finally, DAZ controls pre-meiotic and early meiotic processes in the primate spermatogenesis.

\subsection{Drosophila}

Boule is the only member of the DAZ family genes expressed in Drosophila (Fig. 3). Boule expression is limited to males and its transcripts are absent from flies lacking a germline. Spermatocytes are formed in mutants and fail to undergo meiotic divisions [19-21]. Comparison of the localization of Cyclin A in Boule mutants and wild-type germ cells indicates that the meiotic prophase is normal in Boule mutants while subsequent meiotic stages are aberrant. BOULE protein is present in the nucleus of 
pre-meiotic germ cells, and subsequently moves into the cytoplasm at the beginning of meiosis [22]. The protein is likely required for the efficient translation of Twine, the homolog of the vertebrate $C d c 25$ gene. Cdc25 encodes a phosphatase that activates cell cycle progression by removing phosphate groups from CDC2 which is a Cyclin-dependent kinase that forms heterodimers with Cyclin A and Cyclin B. Although DAZ family proteins may have nuclear functions, in Drosophila BOULE exclusion from the nucleus did not result in a defective phenotype [22].

\begin{tabular}{|c|c|c|c|c|}
\hline Organism & Gene & Mitosis & Meiosis & Differentiation \\
\hline Drosophila & Boule & & & \\
\hline C.elegans & $D a z-1$ & & & \\
\hline Zebrafish & $z D a z l$ & & & \\
\hline Xenopus & XDazl & & & \\
\hline \multirow{2}{*}{ Mouse } & Boule & & & \\
\hline & Dazl & & & \\
\hline \multirow{3}{*}{ Human } & Boule & & & \\
\hline & Dazl & & & \\
\hline & $D A Z$ & & & \\
\hline
\end{tabular}

Figure 3. Expression profile of the DAZ family genes in different organisms.

\subsection{Caenorhabditis}

The Caenorhabditis elegans homologue of Boule is Daz-1 [23]. Daz-1 is expressed in the germ cells of the larval gonads and hermaphrodites. In hermaphrodites, male meiosis and spermatogenesis take place in the L4 larval stage [23]. The germline switches from spermatogenesis to oogenesis (the sperm/oocyte switch) at the transition to the adult stage and produces oocytes thereafter. In contrast, males continue to produce sperm in the adulthood [23]. Loss of Daz-1 function causes sterility in hermaphrodites, by blocking oogenesis at the pachytene stage of meiosis I. Epistasis analysis suggests that this gene exerts its function downstream of Gld-1, the RNA binding protein that in this species governs the early pachytene stage of oogenesis [24]. Spermatogenesis does not appear to be affected in Daz-1 defective males. In fact, deletion of Daz-1 produces sperm fully competent in fertilization [24]. In short, in C. elegans, Daz-1 appears to be crucial for the switch from spermatogenesis to oogenesis but only if the genetic background was conditional masculinization of germline. On the other hand, in C. briggsae, disruption of Daz-1 resulted in a complete loss of the sperm/oocyte switch [23].

\subsection{Zebrafish}

In Zebrafish, a Boule homologue, zDazl was identified (Fig. 3). Maternal zDazl transcripts are localized in the germ plasma of 4-cell stage in Zebrafish $[25,26]$. At the onset of embryogenesis, maternal zDazl transcripts localize at the vegetal pole and migrate toward blastomeres in cytoplasmic streams as early embryogenesis proceeds. In primordial germ cells (PGCs), zDazl antagonizes microRNA (miRNA) -430-mediated repression of the Tdrd7 mRNA which is necessary for the germline specification. Moreover, $z$ Dazl enhances protein synthesis via the $3^{\prime}$-UTR of Dazl mRNA itself and relieves miRNA-mediated repression of germline mRNAs by controlling poly(A) tail length of the target mRNAs [27]. zDazl transcripts are expressed in gonads of both sexes. In the ovary, they are localized in the oocyte cortex.

\subsection{Amphibians}

In the anuran Xenopus laevis, only the expression of the Dazl gene termed as $x$ Dazl has been reported (Fig. 3). While $x D a z l$ transcripts are present in the mitochondrial cloud (source of germinal granule material) of mature oocytes, the protein is detectable in the germ plasma at the early blastula stages in the four blastomeres of the vegetal pole, which give rise to PGCs. In fact, depletion of $x D a z l$ causes a severe reduction or a complete loss of PGCs in tadpoles [28]. In the adult gonads, $x D a z l$ is present in all stages of male and female germ cell development except of mature spermatozoa [29]. In the urodele Axolotl, the other major branch of the amphibian lineage, in which PGCs in the absence of germ plasma are formed by induction, maternal Dazl RNAs are localized in the animal cap and equatorial region of early embryos. At gastrula, neurula, and tailbud embryonic stages, the transcripts are widely distributed and become specifically expressed in PGCs approaching to the gonadal ridges [30].

\subsection{Mouse and Rat}

Mice with Dazl deficiency are infertile, lacking any formation of spermatozoa or oocytes. The insertion of the human DAZ gene into Dazl-/- mouse relieves some of the defects of the male phenotype [31]. DAZL protein is expressed in post-migratory $X X$ and XY PGCs, beginning at about 11.5 days post coitum (dpc) before their sexual differentiation [10]. In Dazl null embryos of inbred genetic mice, $X X$ or XY PGCs migrate to the gonadal ridges but do not develop either male or female features. Instead, they remain in a sexually undifferentiated state, fail to erase and re-establish genomic imprinting and eventually undergo apoptosis [11-13]. Interestingly, in such PGCs, 
DNA methylation regulates negatively the Dazl expression [32]. In supporting a role of Dazl in early stages of germ cell formation, the differentiation of mouse ESCs to PGC-like cells (PGCLCs) was shown to be dependent on Dazl [11]. Ablation of Dazl disrupts a continuum of fundamental genetic and epigenetic events of post-migratory germ cell development both in vivo and in vitro. Also it results in a reduction in post-migratory germ cell numbers, aberrant expression of markers of pluripotency and differentiation, failure to execute nuclear reprogramming and to produce embryonic germ cell (EGC) lines and inability to progress through meiosis [11]. Studies in mice of mixed strain background revealed no defects in $\mathrm{XY} \mathrm{Dazl-/-}$ germ cells at $15.5 \mathrm{dpc}$ [16]. In such mice, spermatogonia remain in the Dazl-/- testis and develop into spermatocytes in a few cases. Ultimately, however, even these cells undergo apoptosis and never develop further at the stage of pachytene spermatocytes [17]. In adult testis, Dazl appears in the nucleus of mitotic spermatogonia, achieving the highest level in the cytoplasm of the pachytene spermatocytes, and decreasing at last stages of spermatogenesis $[16,18]$. In the female, at $13.5 \mathrm{dpc}$, DAZL positive germ cells are present in the ovary, and become homogeneously distributed throughout the organ by $15.5 \mathrm{dpc}$. At $18.5 \mathrm{dpc}$ and postnatal day 0, DAZL positive oocytes are predominantly localized at the periphery of the ovary [14]. DAZL expression persists during oocyte growth and meiotic maturation [15] and is also functional in these stages. Dazl knockdown in mouse GV (germinal vesicle) oocytes leads to defects in oocyte maturation while Dazl knockdown in eggs leads to a defect in oocyte-zygote transition [15]. The late functions of DAZL in oocytes may be conserved in other vertebrates as DAZL is also expressed in late oogenesis in pig and Xenopus [29, 33]. The timing of the loss of female germ cells depends on the genetic background. In fact, in an inbred line of mice, significant loss of germ cells occurred by $14.5 \mathrm{dpc}$. However, in female mice with a mixed strain background, Dazl null PGCs proliferate and enter meiosis normally. There is substantial loss of oocytes only from embryonic $17.5 \mathrm{dpc}$ onward. By postnatal day 4 , there are no oocytes in the ovaries due to failing to progress through meiotic prophase.

Targeted disruption of Boule results in male sterility, due to the arrest of spermatogenesis at the round spermatid stage whereas female are apparently fertile. Thus it leads to the conclusion that Boule is dispensable for mouse oogenesis [34].

In rats, as far as we know, the expression of $D A Z$ gene family has been investigated in the adult testis only. DAZL protein is localized at high level in the cytoplasm of pachytene spermatocyte when the syn- aptonemal complex forms. Subsequently, DAZL disappears in diplotene spermatocytes when chromosomes desynapsed [35].

\subsection{Human}

DAZL was immunolocalised into the nuclei of germ cells (oogonia and prospermatogonia) in $1^{\text {st }}$ trimester gonads of both sexes. At $2^{\text {nd }}$ trimester, in the ovaries, DAZL appears in the cytoplasm of meiotic oocytes, whereas gonocytes with nuclear or cytoplasmic staining were detected throughout the fetal testes. Oocytes within primordial follicles showed low or absent expression of Dazl [34, 36]. By using in situ hybridization, Brkehman et al. [36] reported similar results and observed Dazl expression in granulosa cells curiously. He et al. [14] performed a detailed expression of the DAZL and BOULE proteins in female germ cells during the embryonic and fetal period. They confirmed DAZL expression in oogonia and meiotic oocytes. Moreover, they observed that BOULE is transiently expressed in oocytes at more advanced meiotic stages. However, very rarely they observed germ cells expressing both DAZL and BOULE, suggesting that DAZL and BOULE mark distinct populations of oocytes. DAZL, but not BOULE, remains expressed in oocytes enclosed within primordial follicles. Boule transcripts were reported in the human fetal ovary by two other papers $[34,37]$. Finally, DAZL expression in oocytes at various developmental stages of the adult ovary has been reported [38], while DAZL protein was unusually detected in the cytoplasm of internal theca of the follicles and luteal cells of the corpus luteum [39].

In the male, BOULE was detected in the adult testis, namely in the cytoplasm of pachytene spermatocytes. It persists through meiosis and decreases in early spermatids. DAZ is expressed in spermatogonia, early and late spermatocytes, and postmeiotic germ cells up to sperm [40,41]. Some studies showed that after complete deletion of $D A Z$ genes, sperm production continues but at extremely low levels and rare instances of natural conceptions. This maybe explained in part by some functional overlap between DAZ and Dazl [42-47].

Interestingly, overexpression of Dazl, Boule and $D A Z$ induced both human ES and induced pluripotent stem (iPS) cells to differentiate into PGCLCs, and enhanced their subsequent maturation and progression through meiosis [37, 48], supporting a role of these proteins in PGC specification also in mammals.

\section{Regulation of mRNA translation by DAZ family proteins}

Precursor mRNAs (pre-mRNAs) undergo processing including capping at the $5^{\prime}$ end, $3^{\prime}$ polyad- 
enylation, splicing and editing in some cases to generate mature mRNAs. Following their export to the cytoplasm the distribution, stability and utilization of mRNAs is highly regulated (e.g. by RNA-binding proteins). The post-transcriptional regulation of mRNAs is considered crucial in embryogenesis and gametogenesis. In the embryo, the spatial and temporal regulation of translation of selected mRNAs is frequently achieved by maintaining them in a silent, repressed state until their translation is activated whenever and wherever the encoded protein is needed. During gametogenesis, many genes are transcribed several days before translation occurs, requiring a network of mRNA storage and translational control. The ability of DAZ family members to bind specific mRNAs, typically through their $3^{\prime}$-UTRs, suggests that they may also play roles in controlling mRNA fate in the cytoplasm, with experimental evidence being available in some cases.

As reported above, all three DAZ family proteins possess a highly conserved RRM which is able to bind 3'-UTR sequences of target mRNAs (Fig. 4). Although these proteins are considered as main translation activators, they may exert post-transcriptional regulation at various levels during both germline specification/determination in the embryo and subsequent gametogenesis in both sexes. Interestingly, though DAZ family proteins are predominantly present in the cytoplasm, occasional nuclear localization is also observed. For example, in flies, BOULE protein is initially in the nucleus of early spermatocytes, and then transits to the cytoplasm just before metaphase. Similarly, human and mouse DAZL is nuclear in gonocytes and may translocate from the nuclei of spermatogonia into the cytoplasm of spermatocytes. Some evidence, however, (e.g. see, [22]) suggest that the nucleus is only a storage place of DAZ proteins.

\subsection{Target mRNA for DAZ family proteins}

Several target mRNAs for DAZ family proteins have been identified in different species [49-53]. DAZL has a high-affinity sequence-specific recognition of a GUU triplet in 3'-UTR of mRNAs. It is the RRM motif of DAZL protein that recognizes GUU triplets in the $3^{\prime}$-UTR, which is essential in germ cells development. Mutations of bases within the GUU will severely impair the affinity of binding [53].

In Drosophila, Twine mRNA is translational regulated by BOULE. TWINE protein is a CDC25 phosphatase which is necessary for the G2/M transition in spermatocytes [49]. zDAZL protein specifically binds to the 'GUUC' sequences in the 3'-UTR of the Twine or $z$ Dazl transcripts [52]. Mouse DAZL binds to the consensus (GUn)n in the 5'-UTR of Cdc25C mRNA [50]. Mouse DAZL is found to bind nine known mRNA encoding proteins involved in a variety of cell functions [51]. These include, for example, germ cell adhesion to Sertoli cells (Testis specific-1 (Tpx-1), cytoskeleton assembly (F-actin-capping protein subunit beta, Capp $\beta 1$ ), protein degradation (proteosome a7/C8 subunit, $P \alpha 7 / c 8$ ), translation initiation (G-rich RNA sequence binding factor 1, GRSF1), a TATA box-binding protein (TBP)-like (Telomeric repeats binding site 2, $\operatorname{Tr} f 2$ ). Among these, it includes the mRNA for Cdc25A encoding a threonine/tyrosine phosphatase involved in cell cycle progression, that is identified as the major target of the Drosophila Boule. In addition, Chen et al. confirmed that the increased translation of three mRNAs testis expressed gene 19.1 (Tex19.1), microtubule-associated protein homolog (Tpx2), and Dazl itself was dependent on DAZL protein accumulation (Table 2) [15].

Table 2. Target mRNAs of DAZL protein.

\begin{tabular}{cc}
\hline \multicolumn{2}{c}{ Target mRNAs of DAZL protein } \\
\hline Confirmative targets & Putative targets \\
Tpx 1 1,Pam, GRSF1, Trf2, Capp $\beta 1$, H47, clone & Bub1b, Cdc20, Arid1A, Smarca5 \\
D2, Pa7/c8, Cdc25A, Tex19.1, Dazl itself & \\
\hline
\end{tabular}

Finally, co-immunoprecipitation with DAZL from UV-cross linked mouse testicular extracts identifies the mRNAs encoding mouse VASA homologue $(\mathrm{MVH})$ and a synaptonemal complex component (SYCP3), two proteins playing a pivotal role in germ cell development [54,55].

The Vasa gene also known as $D d x 4$, is originally identified in Drosophila as a maternal-effect gene required for the formation of the abdominal segments and germline specification. It has been found in many other invertebrate and vertebrate species [56]. Analysis of these VASA homologs has revealed a highly conserved role for VASA protein among different organisms, as well as some important differences in its regulation. VASA is an ATP-dependent RNA helicase of the DEAD-box family. In Drosophila it is required for promoting translation of at least two RNAs, Nanos and Gurken, two key determinant mRNA for the fly oogenesis. VASA is localized in the cytoplasm of mouse and human PGCs at early embryonic stages, consistent with the idea that its activity is required for the germline development, likely for preserving pluripotency. In the human, VASA is expressed in migrating PGCs [57], while in the mouse, it is found in PGCs as they arrive into the gonadal ridges. In both species, VASA protein is also expressed in fetal and adult germ cells in both males and females and particularly in spermatocytes and mature oocytes $[57,58]$. SYCP3 is a major component of the lateral elements of the synaptonemal complex of the meiotic germ cells. It 
is initially expressed in both female and male pre-meiotic mouse PGCs, but it becomes subsequently confined to female PGCs entering into meiosis in the fetal ovary [59], and in postnatal meiotic male germs [60]. Vasa and Sycp3 knockout male mice have similar phenotypes with Dazl gene knockout mice [54]. Moreover, the protein levels of SYCP3 and VASA decrease significantly in survival germ cells within testes of Dazl knockout mice [51, 54, 55]. Importantly, DAZL protein increases the translation of the mRNA of both proteins. In vitro, DAZL protein directly binds to the 3'-UTR of Vasa mRNA, and the translation of reporter mRNAs containing the $3^{\prime}$-UTR of Vasa is activated by DAZL in oocyte micro-injection experiments [54]. 3'-UTR of Vasa and Sycp3 contain multiple GUU triplet bases, but the nucleotide sequences in both sides of the GUU are different [53].

\subsection{DAZ family proteins and microRNAs}

MicroRNAs are small non-coding RNAs with a length of 21-23 base pair. Recently, Kedde et al. reported that a germline-specific RNA-binding dead end (DND) protein, suppresses miR-430 function through blocking miRNA accessibility by binding to U-rich mRNA regions (URRs) in zebrafish and human germline cells [61]. Moreover, the loss of DND function or its target sequences make Nanos and Tdrd7 3'-UTRs susceptible to miR-430-mediated repression. In the Zebrafish embryo, miR-430 contributes to re-

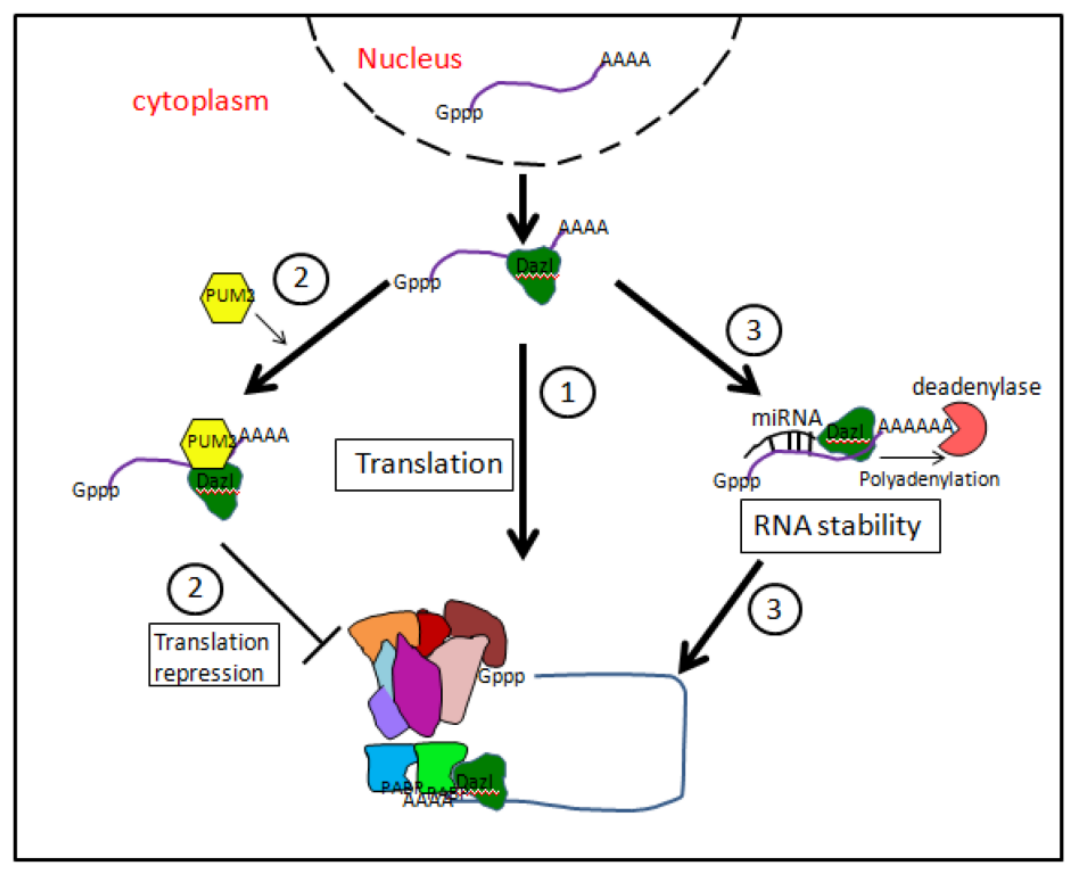

Figure 4. Putative mechanisms of mRNA translation regulation by DAZL proteins. (1) By interacting with translation initiation factor PABPs, DAZL proteins might promote the assembly of $80 \mathrm{~S}$ ribosome. (2) DAZL proteins might interact with PUM2 protein, forming a stable complex able to inhibit mRNA translation. (3) DAZL proteins might favor mRNA polyadenylation by extending poly (A) tail and enhancing mRNA stability. strict Nanos1 and Tudor 7 (Tdrd7) to PGCs by inducing mRNA deadenylation, mRNA degradation, and translational repression of Nanos1 and Tdrd7 mRNAs in somatic cells [62]. DAZL binding to the 3'-UTR can relieve the miR-430-mediated repression of TDRD7, concomitant with an increase in poly(A) tail length [27]. Therefore, through blocking miRNA mediated RNA degradation, Dead end and DAZL help to enable the expression of specific genes in the germline, crucial for the preservation of germline fate (Fig. 4).

\subsection{DAZ family associated proteins}

DAZ family proteins interact with several types of proteins including DAZ associated protein (DAZAP), poly(A)-binding proteins (PABPs), dynein light chain (DLC1), DAZ-interacting protein 1 (DZIP1), and GASZ protein.

Using $D a z$ as bait in a yeast two-hybrid system, two DAZAPs were identified [9]. DAZAP1 encodes a RNA binding protein, which is widely expressed throughout different tissues and is expressed most abundantly in the testis. DAZAP2 encodes a ubiquitously expressed protein with unrecognizable functional motif. DAZAP1 and DAZAP2 binds similarly to both DAZ and DAZL. DAZAP1 may play several roles in the regulation of RNA metabolism [63] and is highly expressed in the mouse testes, predominantly in late stage spermatocytes and post-meiotic spermatids where it likely regulates the splicing of Crem, Crisp2 and Pot1a transcripts involved in male germ cell maturation [64]. DAZAP1 deficiency in mice results in spermatogenic arrest [65]. DAZAP1 is also immulocalized in human luteal cells, granulosa cells and oocytes of the rat ovary. Co-immunoprecipitation experiments show interaction of DAZL protein with DAZAP1 in the ovarian tissues in vivo [66]. DAZAP1, like DAZL, is known to be an mRNA-specific activator of translation, but this appears independent of its ability to bind DAZL, suggesting these functions are separable [67].

In order to exert many of their regulative action, DAZ family proteins often combine with other RBPs [68]. In human germ cells, DAZ and DAZL co-localize with a member of the $P u$ milio family, namely Pumilio RNA-binding family member 2 (PUM2) [69]. Pumilio proteins are usually translation repressors and are known to be needed for maintaining germline stem cells in Drosophila and C. elegans. 
PUM2 forms a stable complex with DAZ and DAZL through both DAZ and RRM motives. Moreover, PUM2 was expressed also in human ES cells [70]. Human BOULE is also able to interact with PUM2 besides forming homodimers [70]. BOULE and PUM2 can form complex on a subset of PUM2 RNA targets distinct from targets bound by PUM2 and DAZL. This suggests that RNA sequences bound by PUM2 may be influenced by protein interactions. The functional role of PUM2/DAZ family member complexes remain unknown but based on the role of Pumilio in translational repression, can be speculated to involved in negatively regulating translation. In this regard, it is mentioned that PUM2 and DAZL are able to bind the same 61 potential RNA targets [71] (Fig. 4).

Evidence exists that DAZL promotes translation initiation on specific mRNAs by interacting with PABPs [72]. These are well-known factors crucial for the translation initiation. PABPs bind the poly (A) tail and contacting factors bound at the $5^{\prime}$ end which effectively circularizes the mRNAs and promotes ribosomal subunit recruitment. Many stored germ cell mRNAs have short poly (A) tails and recruitment of PABPs by DAZL allows PABPs to enhance translation initiation independent of a long poly (A) tail.

DAZL can specifically interact with the DLC1, a component of the dynein-dynactin motor complex. Interaction occurs with the C-terminal end of the DAZL protein. The sub-cellular distribution of DAZL in cell lines is microtubule-dependent and a selected number of DAZL -bound mRNAs could accumulate in the perinuclear area [73]. These suggest that DAZL may function as an mRNA transporter [73]. But at present the functional study of DAZL to transport mRNAs was done in somatic cells and not in germ cells. mRNA transport can contribute to the localized translation of mRNAs. In other words, DAZL may direct the localization of some mRNAs to specific regions within germ cells and/or be involved in the localization of repressed mRNA to sites of mRNA storage where they may be retained in a stable but translationally silent state. Relief of repression may require the loss of repressor proteins (e.g. PUM2) as well as the recruitment of PABPs. Thus it may be speculated that the translational activity of mRNAs bound by DAZL may be affected by their sub-cellular localisation as well as by translational regulatory factors [73]. Dazl is also translocated to stress granules (SGs) upon heat stress. SGs are cytoplasmic particles of eukaryotic cells, which form during cell stress and act as storage sites for repressed mRNAs [74]. Furthermore, stress granules assembly activity is significantly diminished in the early male germ cells of $\mathrm{Dazl}$ knockout mice. The DAZL-containing stress granules seem to play a protective role against heat stress-induced apoptosis by the sequestration of specific signaling molecules, such as receptor for activated C kinase 1 (RACK1), and the subsequent blockage of the apoptotic MAPK pathway [75].

In 2004, Frederick et al. [76] reported the identification of the DZIP (DAZ-Interacting Protein) gene, which encodes at least three different protein isoforms that contain a $\mathrm{C} 2 \mathrm{H} 2$ zinc-finger domain. The DZIP gene is expressed predominantly in human embryonic stem cells and fetal and adult germ cells; moreover, two DZIP protein isoforms colocalized with DAZ and/or DAZL proteins in these tissues. Using co-immunoprecipitation assays, these authors provided also evidence indicating that DZIP may associate with DAZ and its other cofactors in an RNA-binding protein complex that functions in both ES cells and germ cells. The biological role of DZIP1 is, however, still not clearly defined and it has been reported to be involved in the regulation of various molecular processes. The DZIP1 protein is a component of the Hedgehog $(\mathrm{Hh})$ signaling pathway and has a putative regulatory role in Hh signalling and ciliogenesis. Patrícia et al. [77] located DZIP1 protein predominantly in stress granules in the cytoplasm and showed that it is a component of ribonucleoprotein complexes in HeLa cells. Ribonomic analysis of associated mRNAs identified networks of genes involved principally in cell cycle regulation and gene expression.

Wang et al. [78] showed that gain of function of Gasz, a gene previously reported to participate in meiosis of postnatal spermatocytes, led to the most robust upregulation of PGC formation from both human and mouse ESCs. In contrast, Gasz deficiency resulted in pronounced reduction of germ cells during ESC differentiation and decreased expression of VASA and DAZL in gonadal ridges during early embryonic development. Finally, co-immunoprecipitation assays demonstrated that GASZ protein interacted with DAZL, through the protein domain sterile alpha motif (or SAM) and that the GASZ and DAZL complex is able to up-regulate the expression of germ cell genes including Oct4, Stella and Vasa.

\section{Conclusions}

Although the founder cells of the germline are specified differently in invertebrates and vertebrates, several studies have shown that germline specification also in distant species first depends on the global inhibition of mRNA transcription and epigenetic reprogramming in the founder PGCs. After specification, PGCs begin a long journey to form the gametes. They first migrate inside the embryo to join the somatic gonadal cells and subsequently adopt a sexual identity to eventually initiate meiosis and gameto- 
genesis. However, transcriptional repression and epigenetic reprogramming in PGCs are unlikely to be the primary ways used by PGC descendants to regulate gene expression during differentiation. Several lines of evidence suggest that RNA based mechanisms are pivotal in germ cell differentiation. Many of the important regulators of germline development are RNA binding proteins and loss of these factors causes dramatic effects on germline development. DAZ family proteins are central RNA binding proteins in this process. These proteins appear to be associated with common features of germ cells of distant species in both sexes, indicating that unique functions that differentiate germ cells from somatic cells are likely to be conserved in animal species. Both distinctive process of gametogenesis, the specification/determination of germ cells and meiosis, appear controlled by the DAZ family proteins through the post-translation regulation of germline specific proteins at various levels including the transport, stabilization/degradation and transduction of the coding mRNAs. The phylogenetic history of the DAZ family proteins and the multiform ways by which they may regulate post-transcriptional activity of several target mRNAs, likely represent a unique example of regulators of germline development conserved in the evolution.

\section{Acknowledgements}

This work was supported by National Basic Re-
search Program of China (973 Program, 2012CB944401), National Nature Science Foundation (31171376), Program for New Century Excellent Talents in University (NCET-12-1026), the Program of Science and Technology Grant of Qingdao City (12-1-4-5-(10)-jch) and the Taishan Scholar Construction Foundation of Shandong Province.

\section{Competing Interests}

The authors have declared that no competing interest exists.

\section{References}

1. Reijo R, Lee TY, Salo P et al. Diverse spermatogenic defects in humans caused by Y chromosome deletions encompassing a novel RNA-binding protein gene. Nat Genet. 1995; 10: 383-93.

2. Saxena R, Brown LG, Hawkins T et al. The Daz gene cluster on the human $Y$ chromosome arose from an autosomal gene that was transposed, repeatedly amplified and pruned. Nat Genet. 1996; 14: 292-9.

3. Seboun E, Barbaux $\mathrm{S}$, Bourgeron $\mathrm{T}$ et al. Gene sequence, localization, and evolutionary conservation of DAZLA, a candidate male sterility gene. Genomics. 1997; 41: 227-35.

4. Xu EY, Moore FL, Pera RA. A gene family required for human germ cell development evolved from an ancient meiotic gene conserved in metazoans. PNAS. 2001; 98: 7414-9.

5. Teng YN, Lin YM, Lin YH et al. Association of a single-nucleotide polymorphism of the deleted-in-azoospermia-like gene with susceptibility to spermatogenic failure. J Clin Endocr Metab. 2002; 87: 5258-64.

6. David chase cameron sprague. Structure and function of the deleted in azoospermia gene. Texas, USA, Saint Edward's University, 2006.

7. Yen PH, Chai NN, Salido EC. The human Daz genes, a putative male infertility factor on the $\mathrm{Y}$ chromosome, are highly polymorphic in the Daz repeat regions. Mamm Genome. 1997; 8: 756-9.
8. Avis JM, Allain FH, Howe PW et al. Solution structure of the N-terminal RNP domain of U1A protein: the role of C-terminal residues in structure stability and RNA binding. J Mol Biol. 1996; 257: 398-411.

9. Tsui S, Dai T, Roettger S, Schempp W et al. Identification of two novel proteins that interact with germ-cell-specific RNA-binding proteins Daz and DAZL1. Genomics. 2000; 65: 266-73.

10. Seligman J, Page DC. The Dazl gene is expressed in male and female embryonic gonads before germ cell sex differentiation. Biochem Bioph Res Co. 1998; 245: 878-82.

11. Haston KM, Tung JY, Reijo Pera RA. Dazl functions in maintenance of pluripotency and genetic and epigenetic programs of differentiation in mouse primordial germ cells in vivo and in vitro. PloS ONE. 2009; 4: e5654.

12. Gill ME, Hu YC, Lin $Y$ et al. Licensing of gametogenesis, dependent on RNA binding protein DAZL, as a gateway to sexual differentiation of fetal germ cells. PNAS. 2011; 108: 7443-8.

13. Lin Y, Page DC. Dazl deficiency leads to embryonic arrest of germ cell development in XY C57BL/6 mice. Dev Biol. 2005; 288: 309-16.

14. He J, Stewart K, Kinnell HL et al. A developmental stage-specific switch from DAZL to BOLL occurs during fetal oogenesis in humans, but not mice. PloS ONE. 2013; 8: e73996.

15. Chen J, Melton C, Suh N et al. Genome-wide analysis of translation reveals a critical role for deleted in azoospermia-like (Dazl) at the oocyte-to-zygote transition. Gene Dev. 2011; 25: 755-66.

16. Ruggiu M, Speed R, Taggart $\mathrm{M}$ et al. The mouse Dazla gene encodes a cytoplasmic protein essential for gametogenesis. Nature. 1997; 389: 73-7.

17. Schrans-Stassen BH, Saunders PT, Cooke HJ et al. Nature of the spermatogenic arrest in Dazl/- mice. Biol Reprod. 2001; 65: 771-6.

18. Niederberger C, Agulnik AI, Cho $\mathrm{Y}$ et al. In situ hybridization shows that Dazla expression in mouse testis is restricted to premeiotic stages IV-VI of spermatogenesis. Mamm Genome. 1997; 8: 277-8.

19. Castrillon $\mathrm{DH}$, Gonczy $\mathrm{P}$, Alexander $\mathrm{S}$ et al. Toward a molecular genetic analysis of spermatogenesis in Drosophila melanogaster: characterization of male-sterile mutants generated by single P element mutagenesis. Genetics. 1993; 135: 489-505.

20. Eberhart CG, Maines JZ, Wasserman SA. Meiotic cell cycle requirement for a fly homologue of human Deleted in Azoospermia. Nature. 1996; 381: 783-5.

21. Santel A, Winhauer T, Blumer $\mathrm{N}$ et al. The Drosophila don juan (dj) gene encodes a novel sperm specific protein component characterized by an unusual domain of a repetitive amino acid motif. Mech Dev. 1997; 64: 19-30.

22. Cheng MH, Maines JZ, Wasserman SA. Biphasic subcellular localization of the DAZL-related protein boule in Drosophila spermatogenesis. Dev Biol. 1998; 204: 567-76

23. Otori M, Karashima T, Yamamoto M. The Caenorhabditis elegans homologue of deleted in azoospermia is involved in the sperm/oocyte switch. Mol Biol Cell. 2006; 17: 3147-55.

24. Karashima T, Sugimoto A, Yamamoto M. Caenorhabditis elegans homologue of the human azoospermia factor Daz is required for oogenesis but not for spermatogenesis. Development. 2000; 127: 1069-79.

25. Hashimoto $\mathrm{Y}$, Maegawa $\mathrm{S}, \mathrm{Nagai} \mathrm{T}$ et al. Localized maternal factors are required for zebrafish germ cell formation. Dev Biol. 2004; 268: 152-61.

26. Theusch EV, Brown KJ, Pelegri F. Separate pathways of RNA recruitment lead to the compartmentalization of the zebrafish germ plasm. Dev Biol. 2006; 292: 129-41.

27. Takeda $\mathrm{Y}$, Mishima $\mathrm{Y}$, Fujiwara $\mathrm{T}$ et al. DAZL relieves miRNA-mediated repression of germline mRNAs by controlling poly(A) tail length in zebrafish. PloS ONE. 2009; 4: e7513.

28. Houston DW, King ML. A critical role for Xdazl, a germ plasm-localized RNA, in the differentiation of primordial germ cells in Xenopus. Development. 2000; 127: 447-56.

29. Mita K, Yamashita M. Expression of Xenopus Daz-like protein during gametogenesis and embryogenesis. Mech Dev. 2000; 94: 251-5.

30. Bachvarova RF, Masi T, Drum M et al. Gene expression in the axolotl germ line: Axdazl, Axvh, Axoct-4, and Axkit. Dev Dynam. 2004; 231: 871-80.

31. Slee R, Grimes B, Speed RM et al. A human Daz transgene confers partial rescue of the mouse Dazl null phenotype. PNAS. 1999; 96: 8040-5.

32. Maatouk DM, Kellam LD, Mann MR et al. DNA methylation is a primary mechanism for silencing postmigratory primordial germ cell genes in both germ cell and somatic cell lineages. Development. 2006; 133: 3411-8.

33. Liu J, Linher $\mathrm{K}, \mathrm{Li}$ J. Porcine DAZL messenger RNA: its expression and regulation during oocyte maturation. Mol Cell Endocrinol. 2009; 311: 101-8.

34. Shah C, Vangompel MJ, Naeem V et al. Widespread presence of human BOULE homologs among animals and conservation of their ancient reproductive function. PLoS Genet. 2010; 6: e1001022.

35. Rocchietti-March M, Weinbauer GF, Page DC et al. Dazl protein expression in adult rat testis is up-regulated at meiosis and not hormonally regulated. Int J Androl. 2000; 23: 51-6.

36. Brekhman V, Itskovitz-Eldor J, Yodko E et al. The DAZL1 gene is expressed in human male and female embryonic gonads before meiosis. Mol Hum Reprod. 2000; 6: 465-8.

37. Kee $\mathrm{K}$, Angeles VT, Flores $\mathrm{M}$ et al. Human DAZL, DAZ and BOULE genes modulate primordial germ-cell and haploid gamete formation. Nature. 2009; 462: 222-5.

38. Cauffman G, Van de Velde H, Liebaers I et al. DAZL expression in human oocytes, preimplantation embryos and embryonic stem cells. Mol Hum Reprod. 2005 ; 11: 405-11. 
39. Pan HA, Tsai SJ, Chen CW et al. Expression of DAZL protein in the human corpus luteum. Mol Hum Reprod. 2002; 8: 540-5.

40. Habermann B, Mi HF, Edelmann A et al. Daz (Deleted in AZoospermia) genes encode proteins located in human late spermatids and in sperm tails. Hum Reprod. 1998; 13: 363-9.

41. Dorfman DM, Genest DR, Reijo Pera RA. Human DAZL1 encodes a candidate fertility factor in women that localizes to the prenatal and postnatal germ cells. Hum Reprod. 1999; 14: 2531-6.

42. Simoni M, Tüttelmann F, Gromoll J et al. Clinical consequences of microdeletions of the $\mathrm{Y}$ chromosome: the extended Münster experience. Reprod Biomed online. 2008; 16: 289-303.

43. Ferlin A, Arredi B, Speltra E et al. Molecular and clinical of $\mathrm{Y}$ chromosome microdeletions in infertile men: a 10-year experience in Italy. J Clin Endocrinol Metab. 2007; 92: 762-70.

44. Kühnert B, Gromoll J, Kostova E et al. Case report: natural transmission of an AZFc Y-chromosomal microdeletion from father to his sons. Hum Reprod. 2004; 19: 886-8.

45. Xia XY, Cui YX, Pan LJ et al. Analysis of an AZFc deletion family with natural transmission. Zhonghua Nan Ke Xue. 2006; 12: 720-2.

46. Calogero AE, Garofalo MR, Barone $\mathrm{N}$ et al. Spontaneous transmission from a father to his son of a $\mathrm{Y}$ chromosome microdeletion involving the deleted inazoospermia (DAZ) gene. J Endocrinol Invest. 2002; 25: 631-4.

47. Navarro-Costa P, Plancha CE, Gonçalves J. Genetic dissection of the AZF regions of the human $Y$ chromosome: thriller or filler for male (in)fertility? J Biomed Biotechnol. 2010.

48. Medrano JV, Ramathal C, Nguyen HN, Simon C, Reijo Pera RA. Divergent RNA-binding proteins, DAZL and VASA, induce meiotic progression in human germ cells derived in vitro. Stem Cells. 2012; 30: 441-51.

49. Maines JZ, Wasserman SA. Post-transcriptional regulation of the meiotic Cdc25 protein Twine by the Dazl orthologue Boule. Nat Cell Biol. 1999; 1: 171-4.

50. Venables JP, Ruggiu M, Cooke HJ. The RNA-binding specificity of the mouse Dazl protein. Nucleic Acids Res. 2001; 29: 2479-83.

51. Jiao X, Trifillis P, Kiledjian M. Identification of target messenger RNA substrates for the murine deleted in azoospermia-like RNA-binding protein. Biol Reprod. 2002; 66: 475-85.

52. Maegawa S, Yamashita M, Yasuda K et al. Zebrafish Daz-like protein controls translation via the sequence 'GUUC'. Genes Cells. 2002; 7: 971-84.

53. Jenkins HT, Malkova B, Edwards TA. Kinked beta-strands mediate high-affinity recognition of mRNA targets by the germ-cell regulator DAZL. PNAS. 2011; 108: 18266-71.

54. Reynolds N, Collier B, Maratou K et al. Dazl binds in vivo to specific transcripts and can regulate the pre-meiotic translation of Mvh in germ cells. Hum Mol Genet. 2005; 14: 3899-909.

55. Reynolds N, Collier B, Bingham V et al. Translation of the synaptonemal complex component Sycp3 is enhanced in vivo by the germ cell specific regulator Dazl. RNA. 2007; 13: 974-81.

56. Raz E. The function and regulation of vasa-like genes in germ-cell development. Genome Biol. 2000; 1: 1017.

57. Castrillon DH, Quade BJ, Wang TY et al. The human VASA gene is specifically expressed in the germ cell lineage. PNAS. 2000; 97: 9585-90.

58. Toyooka $Y$, Tsunekawa N, Takahashi $Y$ et al. Expression and intracellular localization of mouse Vasa-homologue protein during germ cell development. Mech Dev. 2000; 93: 139-49.

59. Di Carlo AD, Travia G, De Felici M. The meiotic specific synaptonemal complex protein SCP3 is expressed by female and male primordial germ cells of the mouse embryo. Int J Dev Biol. 2000; 44: 241-4.

60. Yuan L, Liu JG, Zhao J et al. The murine SCP3 gene is required for synaptonemal complex assembly, chromosome synapsis, and male fertility. Mol Cell. 2000; 5: 73-83.

61. Kedde M, Strasser MJ, Boldajipour B et al. RNA-binding protein Dnd1 inhibits microRNA access to target mRNA. Cell. 2007; 131: 1273-86.

62. Mishima Y, Giraldez AJ, Takeda Y et al. Differential regulation of germline mRNAs in soma and germ cells by zebrafish miR-430. Curr Biol. 2006; 16: 2135-42.

63. Yang HT, Peggie M, Cohen P et al. DAZAP1 interacts via its RNA-recognition motifs with the C-termini of other RNA-binding proteins. Biochem Bioph Res Co. 2009; 380: 705-9.

64. Chen HY, Yu YH, Yen PH. DAZAP1 regulates the splicing of Crem, Crisp2 and Pot1a transcripts. Nucleic Acids Res. 2013; 41: 9858-69.

65. Hsu LC, Chen HY, Lin YW et al. DAZAP1, an hnRNP protein, is required for normal growth and spermatogenesis in mice. RNA. 2008; 14: 1814-22.

66. Pan HA, Lin YS, Lee KH et al. Expression patterns of the Daz-associated protein DAZAP1 in rat and human ovaries. Fertil Steril. 2005; 2: 1089-94.

67. Smith RW, Anderson RC, Smith JW et al. DAZAP1, an RNA-binding protein required for development and spermatogenesis, can regulate mRNA translation. RNA. 2011; 17: 1282-95.

68. Yen PH. Putative biological functions of the Daz family. Int J Androl. 2004; 27: $125-9$.

69. Moore FL, Jaruzelska J, Fox MS et al. Human Pumilio-2 is expressed in embryonic stem cells and germ cells and interacts with Daz (Deleted in AZoospermia) and Daz-like proteins. PNAS. 2003; 100: 538-43.

70. Urano J, Fox MS, Reijo Pera RA. Interaction of the conserved meiotic regulators, BOULE (BOL) and PUMILIO-2 (PUM2). Mol Reprod Dev. 2003; 71: 290-8.
71. Fox M, Urano J, Reijo Pera RA. Identification and characterization of RNA sequences to which human PUMILIO-2 (PUM2) and deleted in Azoospermia-like (DAZL) bind. Genomics. 2005; 85: 92-105.

72. Collier B, Gorgoni B, Loveridge $\mathrm{C}$ et al. The DAZL family proteins are PABP-binding proteins that regulate translation in germ cells. EMBO J. 2005; 24: 2656-66.

73. Lee KH, Lee S, Kim B et al. Dazl can bind to dynein motor complex and may play a role in transport of specific mRNAs. EMBO J. 2006; 25: 4263-70.

74. Kedersha N, Anderson P. Stress granules: sites of mRNA triage that regulate mRNA stability and translatability. Biochem Soc Trans. 2002; 30: 963-9.

75. Kim B, Cooke HJ, Rhee K. DAZL is essential for stress granule formation implicated in germ cell survival upon heat stress. Development. 2012; 139: 568-78.

76. Frederick L M, Jadwiga J, David MD et al. Identification of a novel gene, DZIP (Daz-interacting protein), that encodes a protein that interacts with Daz (deleted in azoospermia) and is expressed in embryonic stem cells and germ cells. Genomics. 2004; 83: 834-43.

77. Patrícia S, Sotelo-Silveira J, Stimamiglio MA et al. Ribonomic analysis of human DZIP1 reveals its involvement in ribonucleoprotein complexes and stress granules. BMC Mol Biol. 2014; doi: 10.1186/1471-2199-15-12.

78. Wang $\mathrm{Q}$, Liu $\mathrm{X}$, Tang $\mathrm{N}$ et al. GASZ promotes germ cell derivation from embryonic stem cells. Stem Cell Res. 2013; 11: 845-60. 